\title{
Metastasectomy of oligometastatic urothelial cancer: a single-center experience
}

\author{
Tim Muilwijk ${ }^{1 \#}$, Murat Akand ${ }^{1,2 \#}$, Frank Van der $\mathrm{Aa}^{1}$, Herlinde Dumez ${ }^{3}$, Gert De Meerleer ${ }^{4}$, \\ Dirk Van Raemdonck ${ }^{5}$, Paul De Leyn ${ }^{5}$, Hendrik Van Poppel ${ }^{1}$, Maarten Albersen $^{1}$, Steven Joniau ${ }^{1}$ \\ ${ }^{1}$ Department of Urology, University Hospitals Leuven, Leuven, Belgium; ${ }^{2}$ School of Medicine, Department of Urology, Selçuk University, Konya, \\ Turkey; ${ }^{3}$ Department of General Medical Oncology, ${ }^{4}$ Department of Radiation Oncology, ${ }^{5}$ Department of Thoracic Surgery, University Hospitals \\ Leuven, Leuven, Belgium \\ Contributions: (I) Conception and design: T Muilwijk, M Akand, S Joniau; (II) Administrative support: None; (III) Provision of study materials \\ or patients: F Van der Aa, H Dumez, G De Meerleer, D Van Raemdonck, P De Leyn, H Van Poppel, M Albersen, S Joniau; (IV) Collection and \\ assembly of data: T Muilwijk, M Akand; (V) Data analysis and interpretation: T Muilwijk, M Akand, S Joniau; (VI) Manuscript writing: All authors; \\ (VII) Final approval of manuscript: All authors. \\ \#These authors contributed equally to this work. \\ Correspondence to: Tim Muilwijk. Department of Urology, University Hospitals Leuven, Herestraat 49, 3000 Leuven, Belgium. \\ Email: tim.muilwijk@uzleuven.be.
}

Background: Survival in patients with urothelial cancer (UC) recurrence after initial treatment with curative intent is limited and treatment options are sparse. Metastasectomy could be considered a treatment option in selected cases. Identifying prognostic factors for survival can be used to counsel patients and aid multidisciplinary teams in making treatment decisions.

Methods: We collected a retrospective case series of patients undergoing metastasectomy for oligometastatic UC between 1999 and 2018 at University Hospitals Leuven. Oligometastatic UC was defined as recurrence of UC in a single organ with $\leq 3$ metastases. Survival outcomes of interest were: overall survival (OS), cancer-specific survival (CSS), and secondary recurrence-free survival (RFS2). Complications were reported using the Clavien-Dindo classification (CDC). Survival analysis are descriptive and were performed using Kaplan-Meier plots to visualize survival data and log-rank was used to compare survival between groups.

Results: From 1999 to 2018, a total of 22 patients underwent metastasectomy of oligometastatic UC. Metastasectomy sites were: pulmonary (59.1\%), loco-regional (13.6\%), hepatic (9.1\%), adrenal (4.5\%), testicular (4.5\%), nodal above aortic bifurcation (4.5\%), and renal transplant (4.5\%). The 5-year OS, CSS and RFS2 after metastasectomy were $51.4 \%, 57.0 \%$, and $49.9 \%$, respectively. Patients with primary upper tract urothelial cancer (UTUC) involvement and patients treated with hepatic metastasectomy had a significantly worse OS, CSS, and RFS2. Patients with a lesion size $>8 \mathrm{~mm}$ and patients with $>1$ pulmonary lesion had a significantly worse CSS. Two CDC grade 3B occurred during follow-up and were both nonprocedure related.

Conclusions: Metastasectomy of oligometastatic UC is feasible and can achieve durable cancer control in a highly selected subgroup of patients. Our results suggest that patients with hepatic metastases or primary UTUC involvement could be considered poor candidates for metastasectomy, while patients with a small $(<8 \mathrm{~mm})$ or solitary pulmonary lesion might benefit most. These findings should be validated in multiinstitutional collaborations or prospective clinical studies.

Keywords: Urothelial cancer (UC); metastatic urothelial cancer (metastatic UC); recurrence; oligometastatic; solitary metastasis; surgery; metastasectomy

Submitted Oct 16, 2019. Accepted for publication Feb 14, 2020.

doi: $10.21037 /$ tau-19-624

View this article at: http://dx.doi.org/10.21037/tau-19-624 


\section{Introduction}

Urothelial cancer (UC) is the most common malignancy of the urinary tract and the ninth most common cancer with 510.000 new cases diagnosed worldwide in 2015 (1). After treatment for muscle-invasive bladder cancer (BC) by radical cystectomy, around $35 \%$ of patients develop local or distant recurrence, resulting in a 5 -year recurrencefree survival (RFS) of 58.3-68.0\%. Median cancer-specific survival (CSS) after recurrence is only 6.9 months $(2,3)$. Over two-thirds of patients with UC recurrence after radical cystectomy die within 12 months (3).

Treatment options are sparse for patients facing UC recurrence. First-line systemic platinum-based combination chemotherapy (CHT) is standard of care, with a limited 5-year overall survival (OS) of 13-17\% (4). Second-line immune checkpoint inhibitors (ICIs) — or first-line in case of cisplatin-ineligibility-are more recent treatment options for patients with UC recurrence. Overall response rates (ORRs) of ICIs are 15-21\% with durable responses only seen in some patients (5).

Metastasis-directed therapy (MDT) is common practice in different fields of oncology. Some patients with limited $\mathrm{UC}$ recurrence, also referred to as oligometastatic UC, could be considered candidates for MDT by surgical resection or targeted radiotherapy. Retrospective uncontrolled series, limited by low granulation in data, suggest a benefit of surgical removal of UC recurrence in highly selected patients, mostly in combination with systemic CHT (6-8). We aimed to identify prognostic factors for OS, CSS, and secondary RFS (RFS2) after metastasectomy for patients with oligometastatic UC in a single high-volume center.

\section{Methods}

We queried the UC registry of the University Hospitals Leuven from 1999 to 2018 after approval of the Ethics Committee Research UZ/KU Leuven (local study number S59188) to identify patients with metastasectomy for oligometastatic UC. The following search terms were used: "urothelial cancer" (and synonyms) in combination with "metastasis", "metastases", "oligometastic" or "solitary metastasis". A retrospectively chart review was performed by a doctoral researcher (TM) who was not involved in the treatment. Patients were identified by reviewing admission notes and surgery reports of the Departments of Urology and Thoracic Surgery. We defined oligometastatic UC as recurrence of $\mathrm{UC}$ in a single organ with $\leq 3$ metastases.
Inclusion criteria were initial treatment with curative intent with pathology-proven UC, metastasectomy with curative intent of metastasis in a single organ with $\leq 3$ metastases, and good performance status (ECOG $\leq 2)$. Exclusion criteria were palliative resections without curative intent, duration of follow-up < 90 days, and inconsistency between pathology of the primary tumor and the metastasis.

Data on the following characteristics were retrospectively collected: age, gender, body-mass index (BMI), smoking status, American Society of Anesthesiologists (ASA) physical status classification (as assessed by anesthesiologist), Eastern Cooperative Oncology Group (ECOG) score, localization of primary tumor(s), history of $\mathrm{BC}$, history of upper tract urothelial cancer (UTUC), previous cyst(-oprostat-)ectomy, previous nephro-ureterectomy, clinical and pathological TNM (tumor-node-metastasis) classification, presence of carcinoma in situ (CIS), presence of lymphovascular invasion (LVI), presence of variant histology, surgical margin status, administration of adjuvant or neoadjuvant CHT and/or radiotherapy in the context of the primary tumor treatment and/or the metastasectomy, size of the metastasis, number of metastases, and complications. Complications were scored using the Clavien-Dindo classification (CDC) following the European Association of Urology (EAU) Guidelines recommendations $(9,10)$ and timing of postoperative complications was scored using $<30,30-90$, or $>90$ days interval from metastasectomy. The following survival outcomes were evaluated: OS, CSS and RFS2.

Follow-up was performed every 3 months for 2 years, then every 6 months for 3 years and annually thereafter. We defined loco-regional recurrence as recurrence within the cystectomy bed and/or the pelvic lymph node template. Distant metastases were defined as lymph nodes above the aortic bifurcation for BC, non-regional lymph nodes for UTUC, bone metastases and/or visceral metastases.

Analyses were performed in $\mathrm{R}$ (version 3.4.3) using the 'dplyr', 'survival', and 'survminer' packages. Summary statistics were presented for continuous variables as median with interquartile range (IQR) and for categorical variables as frequencies and proportions. Kaplan-Meier plots were used to visualize survival data. Log-rank was used to compare survival between groups.

\section{Results}

\section{Patient characteristics}

After applying inclusion and exclusion criteria (Figure 1), 


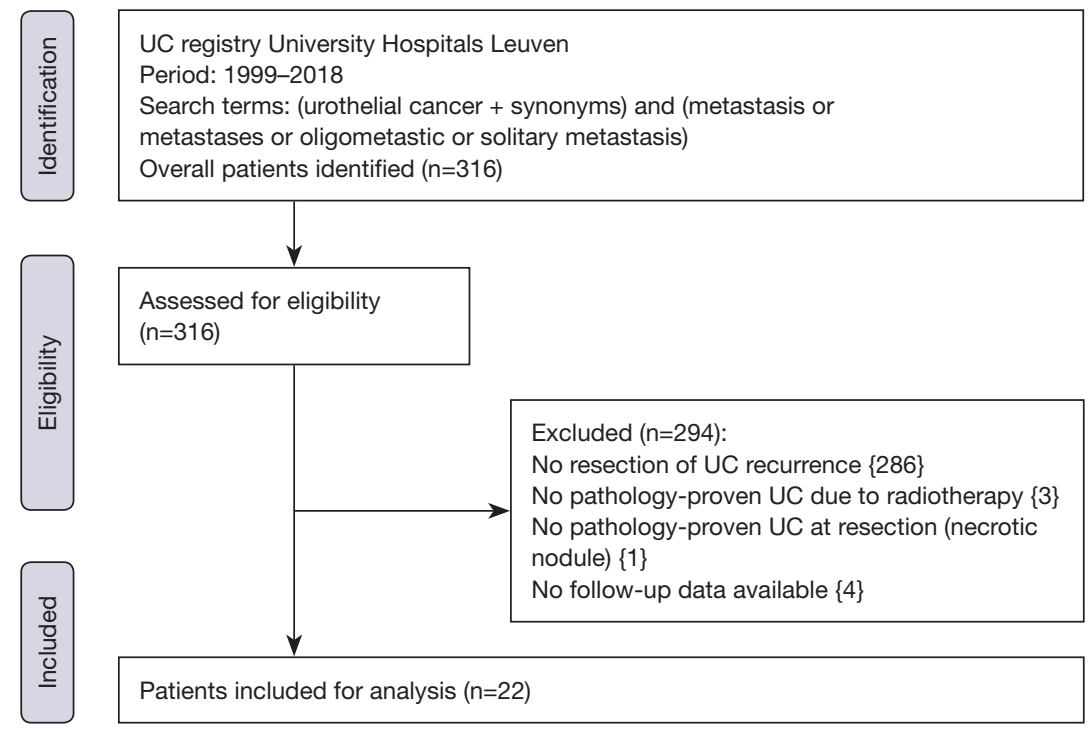

Figure 1 Flow diagram of patients' selection process.

we identified a total of 22 patients whom underwent metastasectomy for UC recurrence with a median interval between primary surgery with curative intent and metastasectomy of 28 months (IQR: 11.1-48.6). Median follow-up after metastasectomy was 24.9 months (IQR: 11.9-82.1) with a minimum follow-up of 4.0 months. Clinical and pathological characteristics, locations of UC recurrences, and type of primary surgery are summarized in Table 1. Nineteen patients (86.3\%) developed metastasis following radical surgery, while 3 patients $(13.6 \%$ ) developed a metastasis following transurethral resection of bladder tumor (TURBT): 1 patient with pTa BC and endoscopically treated pTa UTUC, 1 patient with pT1 BC without UTUC, and 1 patient with pT2 BC without UTUC who was diagnosed with a solitary lung lesion 20 days after TURBT and received radical treatment after the metastasectomy. Neoadjuvant CHT (GemcitabineCisplatin) was administered to 3 patients who underwent radical cystectomy as primary treatment. CHT was administered to 2 patients before undergoing metastasectomy: in 1 patient with liver metastasis and 1 patient with testicular metastasis. Both patients had good response to CHT and metastasectomy was performed as consolidation therapy. Other reasons to opt for metastasectomy were: histopathological confirmation of metastatic disease $(68.2 \%)$, resection of limited locoregional recurrence $(13.7 \%)$, surgery due to intolerance to $\mathrm{CHT}(4.5 \%)$, and resection of tumoral seeding in an afunctional renal transplant (4.5\%).

\section{Survival in all patients}

The 5-year OS, CSS and RFS2 after metastasectomy were 51.4\% [95\% confidence interval (CI): 32.4-81.4], 57.0\% (95\% CI: $36.8-88.2$ ), and 49.9\% (95\% CI: 30.9-80.7), respectively; with a median OS and RFS2 of 98.2 months [95\% CI: 23.4-not reached (NR)] and 33.3 months (95\% CI: 11.4-NR) (OS: Figure 2). The median CSS was NR. Figure 3 illustrates the therapeutic flow of all patients after their metastasectomy. Twelve patients (54.5\%) remained recurrence-free and had a median OS of 154.0 months (95\% CI: 98.2-NR). Ten patients (45.5\%) had a secondary recurrence after a median RFS2 of 9.1 months $(95 \% \mathrm{CI}$ : $5.8-\mathrm{NR})$ and had an OS of 24.7 months (13.5-NR). Three of these patients were treated with MDT ( 2 with secondary metastasectomy and 1 with radiotherapy) and remained recurrence-free after a median follow-up of 73.6 months (IQR: 39.6-86.8). The other 7 patients developed polymetastastic disease and died with a median OS from metastasectomy of 7.6 months (95\% CI: 3.8-NR). Table 2 provides an overview of median time to secondary metastasis after metastasectomy stratified by primary metastasectomy site or by secondary metastasis site. We performed a separate analysis of patients whom did not receive $\mathrm{CHT}$ prior to metastasectomy $(\mathrm{n}=20)$ with a median OS of 98.2 months (IQR: 23.4-NR; Figure S1), median 
Table 1 Clinical and pathological characteristics of all patients

\begin{tabular}{|c|c|c|}
\hline Characteristics & No. & $\%$ \\
\hline Total & 22 & 100.0 \\
\hline \multicolumn{3}{|l|}{ Gender } \\
\hline Male & 21 & 95.5 \\
\hline Female & 1 & 4.5 \\
\hline \multicolumn{3}{|l|}{ Age at ..., years } \\
\hline Primary treatment, IQR & 68.2 & $60.2-73.1$ \\
\hline UC recurrence, IQR & 70.6 & $61.9-74.9$ \\
\hline \multicolumn{3}{|l|}{ Time to UC recurrence, months } \\
\hline IQR & 28 & $11.1-48.6$ \\
\hline \multicolumn{3}{|l|}{ ASA score } \\
\hline 0 & 0 & 0 \\
\hline 1 & 2 & 9.1 \\
\hline 2 & 14 & 63.6 \\
\hline 3 & 5 & 22.7 \\
\hline 4 & 1 & 4.5 \\
\hline 5 & 0 & 0 \\
\hline \multicolumn{3}{|l|}{ ECOG score } \\
\hline 0 & 3 & 13.6 \\
\hline 1 & 16 & 72.7 \\
\hline 2 & 3 & 13.6 \\
\hline \multicolumn{3}{|l|}{ Metastasis site } \\
\hline Pulmonary & 13 & 59.1 \\
\hline Loco-regional & 3 & 13.6 \\
\hline Hepatic & 2 & 9.1 \\
\hline Adrenal & 1 & 4.5 \\
\hline Testicular & 1 & 4.5 \\
\hline Nodal above aortic bifurcation & 1 & 4.5 \\
\hline Renal transplant & 1 & 4.5 \\
\hline $\mathrm{CHT}$ prior to metastasectomy & 2 & 9.1 \\
\hline \multicolumn{3}{|l|}{ Primary treatment } \\
\hline Cystoprostatectomy & 14 & 63.6 \\
\hline Nephro-ureterectomy & 3 & 13.6 \\
\hline Maximal TURBT & 3 & 13.6 \\
\hline Complete urinary tract extirpation & 1 & 4.5 \\
\hline Pelvic exenteration & 1 & 4.5 \\
\hline
\end{tabular}

Table 1 (continued)
Table 1 (continued)

\begin{tabular}{|c|c|c|}
\hline Characteristics & No. & $\%$ \\
\hline Neoadjuvant $\mathrm{CHT}$ primary & 3 & 13.6 \\
\hline \multicolumn{3}{|l|}{$B C$ staging } \\
\hline Total & 20 & 100.0 \\
\hline \multicolumn{3}{|l|}{ pT stage } \\
\hline T1 & 8 & 40.0 \\
\hline$\geq \mathrm{T} 2$ & 12 & 60.0 \\
\hline \multicolumn{3}{|l|}{ pN stage } \\
\hline No & 15 & 75.0 \\
\hline $\mathrm{N}+$ & 2 & 10.0 \\
\hline $\mathrm{Nx}$ & 3 & 15.0 \\
\hline \multicolumn{3}{|l|}{ UTUC staging } \\
\hline Total & 6 & 100.0 \\
\hline \multicolumn{3}{|l|}{ pT stage } \\
\hline $\mathrm{T} 1$ & 5 & 83.3 \\
\hline$\geq \mathrm{T} 2$ & 1 & 16.7 \\
\hline \multicolumn{3}{|l|}{$\mathrm{pN}$ stage } \\
\hline No & 2 & 33.3 \\
\hline $\mathrm{N}_{+}$ & 3 & 50.0 \\
\hline$N x$ & 1 & 16.7 \\
\hline
\end{tabular}

UC, urothelial cancer; IQR, interquartile range; ASA score, American Society of Anesthesiologists score; BC, bladder cancer; ECOG, Eastern Cooperative Oncology Group; CHT, chemotherapy; UTUC, upper urothelial tract cancer; pT stage, pathological tumor stage; pN stage, pathological nodal stage.

CSS NR, and RFS2 of 104.7 months (23.8-NR).

\section{Survival after pulmonary metastasectomy}

The median OS of patients after pulmonary metastasectomy $(\mathrm{n}=13)$ was 154.0 months (95\% CI: 23.4-NR) with a 5 -year OS of $55.9 \%$ (95\% CI: 33.2-94.3). The 5-year OS and CSS for patients $(\mathrm{n}=8)$ with a solitary pulmonary lesion were 87.5\% (95\% CI: 67.3-100) and 100\% (95\% CI: NR). Figure 4 illustrates the therapeutic flow of patients with pulmonary metastasis-only $(n=13)$. Number of pulmonary lesions were: 1 lesion in 8 patients, 2 lesions in 4 patients, and 3 lesions in 1 patient. A total of 8 patients with pulmonary metastases remained recurrence-free after metastasectomy after a median follow-up of 23.3 months (95\% CI: 12.1-91.5). 

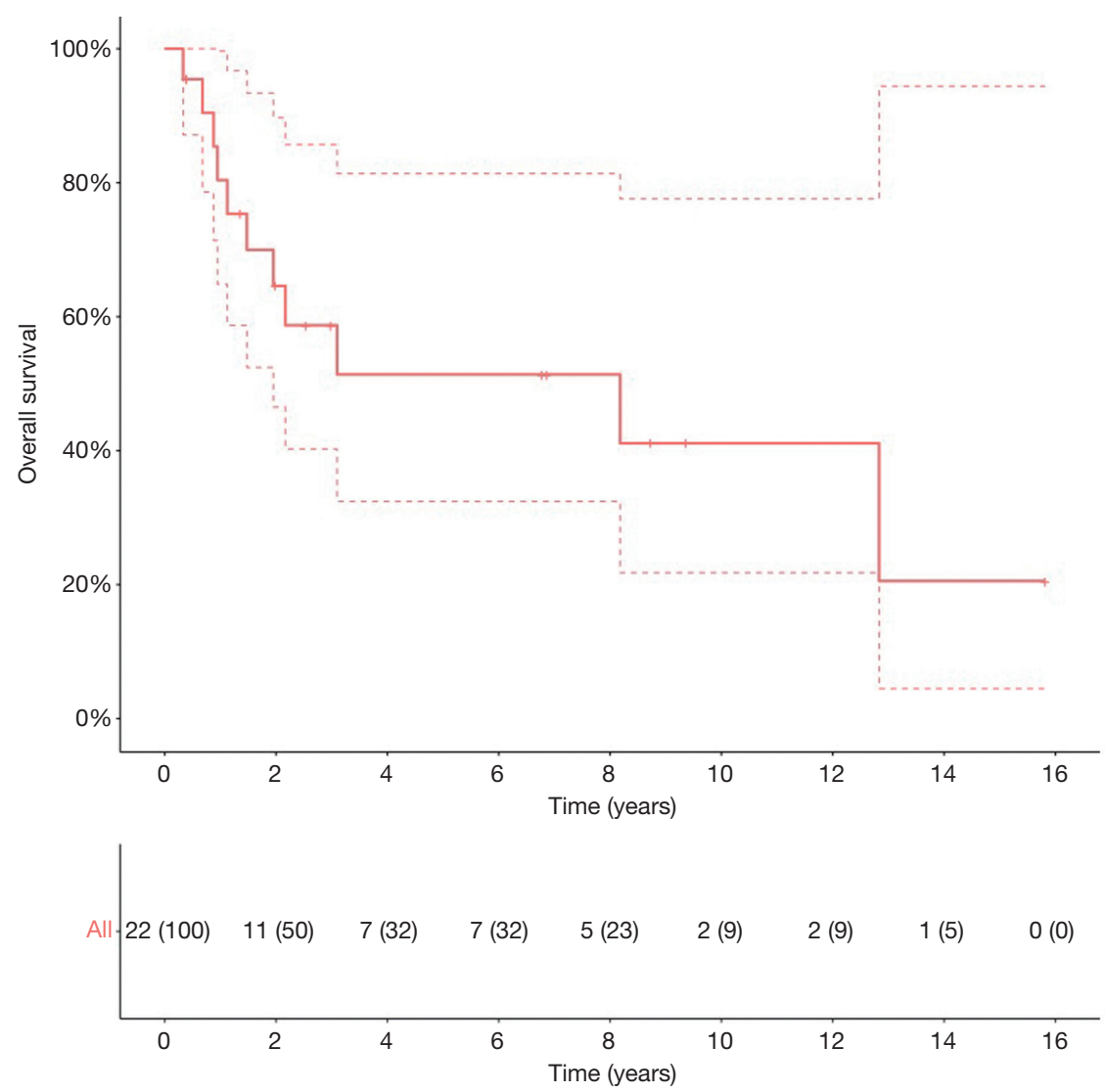

Figure 2 OS of all patients: the 5-year OS was 51.4\% (95\% CI: 32.4-81.4) with a median OS of 98.2 months (95\% CI: 23.4-NR). Dotted lines are the CI. OS, overall survival; CI, confidence interval; NR, not reached.

Five patients with a pulmonary metastasis had a secondary recurrence after metastasectomy with a median OS from metastasectomy of 26.0 months (IQR: 23.4-NR). Two of these patients had a solitary lesion that was treated with a secondary metastasectomy; the two patients remained recurrence-free with a median OS from secondary recurrence of 73.6 months (IQR: 40.0-100.1). The other 3 patients developed polymetastatic disease with a median OS from secondary recurrence of 10.7 months (95\% CI: 4.8-NR).

\section{Prognostic variables}

Patients with primary UTUC involvement $(n=6)$ and patients treated with hepatic metastasectomy $(n=2)$ had significant worse $\mathrm{OS}(\mathrm{P}=0.005 ; \mathrm{P}=0.04)$, CSS $(\mathrm{P}=0.001$; $\mathrm{P}=0.002)$ and RFS2 $(\mathrm{P}=0.001 ; \mathrm{P}=0.02)$ (Figure S2). The 2 patients with hepatic metastasectomies both had primary UTUC involvement. In a separate analysis, excluding the 2 patients with hepatic metastasis, patients with primary
UTUC involvement also had significantly worse CSS $(\mathrm{P}=0.03)$, but no significant difference in OS and RFS2. Patients with a lesion size $>8 \mathrm{~mm}$ had significantly worse CSS compared to $\leq 8 \mathrm{~mm}(\mathrm{P}=0.05)$ (Figure S3). Patients with pulmonary metastasis had significantly worse CSS in case of $>1$ lesion compared to 1 lesion $(\mathrm{P}=0.02)$ (Figure S4).

\section{Complications}

Table 3 provides an overview of all complications after metastasectomy with their CDC grade. A total of 12 complications occurred during 10 procedures (41.7\%). Two grade 1 complications $(8.3 \%$ ) and 8 grade 2 complications (29.4\%) occurred. Two complications (4.2\%) were grade $3 \mathrm{~B}$ and non-procedure related, which occurred after the same procedure $\leq 30$ days from metastasectomy: a computed tomography (CT)-guided percutaneous drainage of a cholecystitis complicated by a hemothorax after removal of the drainage tube of the gallbladder. The hemothorax 


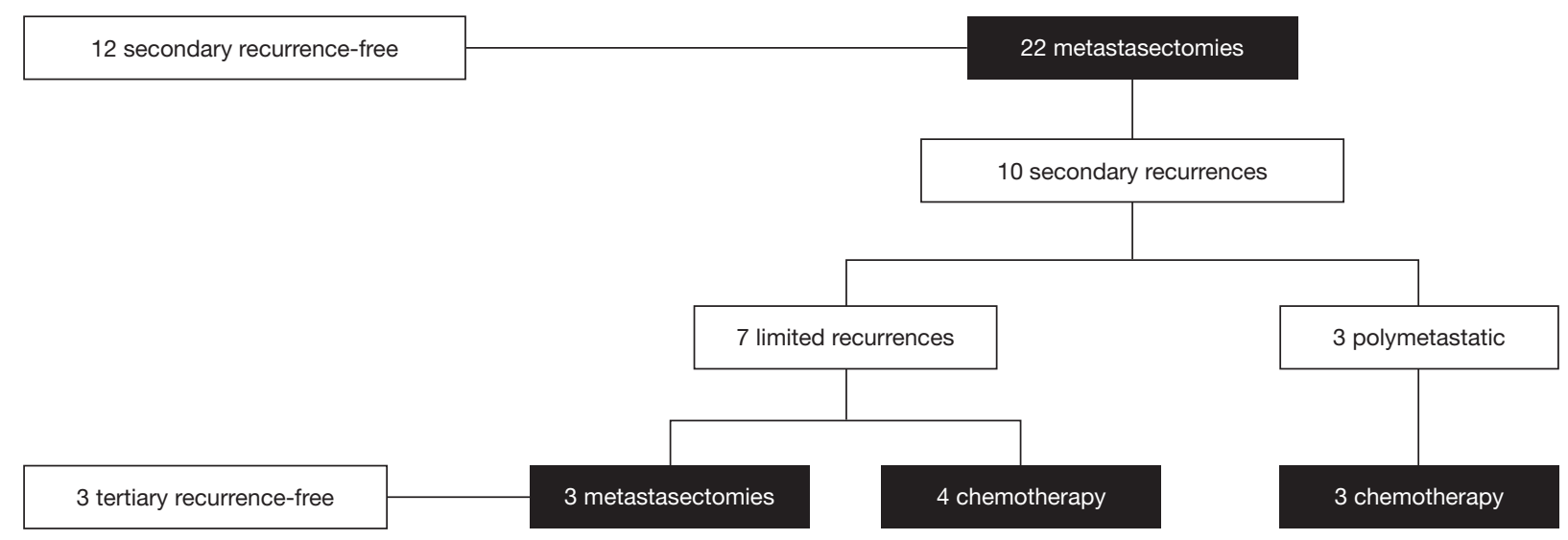

Figure 3 Therapeutic flow of all patients undergoing metastasectomy $(\mathrm{n}=22)$.

Table 2 Median time to secondary metastasis after metastasectomy stratified by primary metastasectomy site or by secondary metastasis site

\begin{tabular}{|c|c|c|c|c|}
\hline Median time to secondary metastasis stratified by... & No. & $\%$ & Months & IQR \\
\hline \multicolumn{5}{|l|}{ Primary metastasectomy site } \\
\hline Lung & 5 & 20.0 & 7.0 & $5.7-11.2$ \\
\hline Liver & 2 & 10.0 & 4.6 & $4.0-5.2$ \\
\hline Nodal above aortic bifurcation (BC) & 1 & 30.0 & 23.8 & NA \\
\hline Renal transplant & 1 & 10.0 & 11.4 & NA \\
\hline \multicolumn{5}{|l|}{ Secondary metastasis site } \\
\hline Polymetastatic & 3 & 30.0 & 7.0 & $5.1-9.1$ \\
\hline Nodal above aortic bifurcation (BC) & 1 & 10.0 & 5.7 & NA \\
\hline Hepatic & 1 & 10.0 & 5.9 & NA \\
\hline
\end{tabular}

$\%$, percentage of patients that had a secondary metastasis. BC, bladder cancer; IQR, interquartile range; NA, not applicable.

was treated with a pulmonary decortication and chest tube placement. Table $S 1$ provides an overview of all procedures and the number of complications per type of procedure. No patients were readmitted, and all reported complications occurred during the initial admission. There were no reoperations and there was no procedure-related mortality.

\section{Discussion}

We report a single-center series of 22 patients with oligometastatic UC who were treated with metastasectomy with a 5 -year OS of $51.4 \%$. In this study, we identified several clinical variables that were prognostic for survival. First, our results suggest that patients with primary UTUC involvement or with hepatic metastasis have significant worse OS, CSS, and RFS2. Patients with a lesion size $>8 \mathrm{~mm}$ demonstrated significant worse CSS. Second, patients who underwent a pulmonary metastasectomy had a long median OS of 154.0 months and a 5 -year OS of $55.9 \%$. Third, patients who underwent a pulmonary metastasectomy had a significantly worse CSS if they had $>1$ lesion. Patients with a solitary pulmonary lesion had a 5 -year 


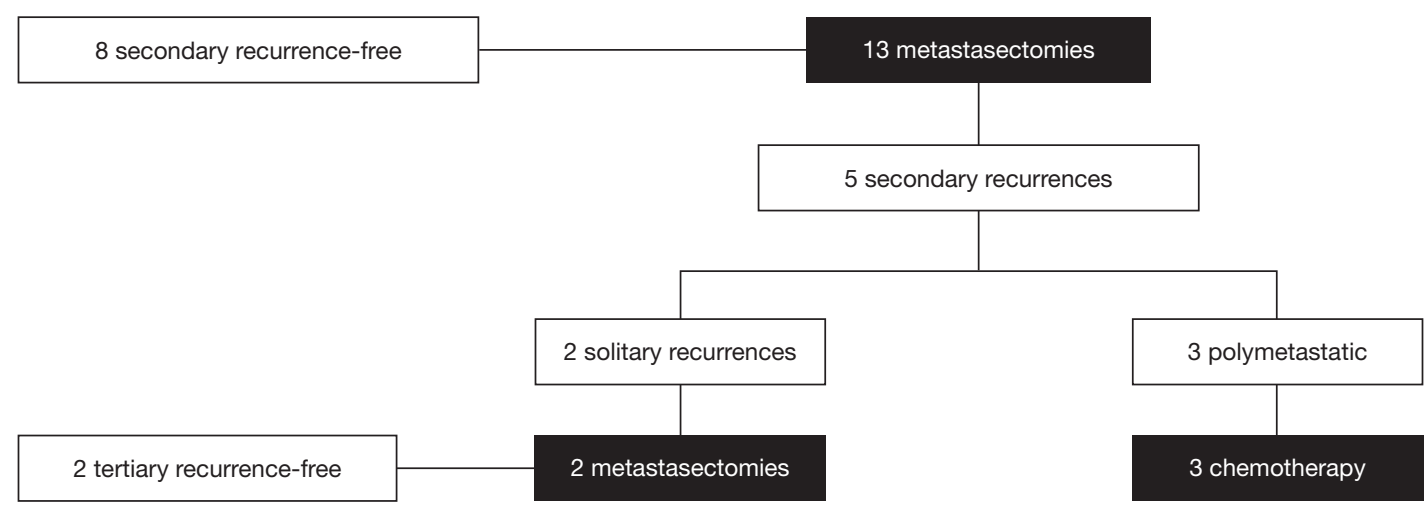

Figure 4 Therapeutic flow of patients undergoing a pulmonary metastasectomy $(\mathrm{n}=13)$.

Table 3 Complications of all metastasectomy procedures $(\mathrm{n}=24)$

\begin{tabular}{|c|c|c|c|c|c|}
\hline CDC & Type & Onset (days) & Procedure-related & No. & $\%$ \\
\hline 1 & Skin & $\leq 30$ & - & 1 & 4.2 \\
\hline \multirow[t]{5}{*}{2} & Gastrointestinal & $\leq 30$ & + & 3 & 12.5 \\
\hline & Neurologic & $\leq 30$ & + & 1 & 4.2 \\
\hline & Infection & $\leq 30$ & $+/-$ & 1 & 4.2 \\
\hline & Skin & $>30-\leq 90$ & + & 1 & 4.2 \\
\hline & Infection & $>30-\leq 90$ & - & 1 & 4.2 \\
\hline $3 B$ & Gastrointestinal $^{\#}$ & $\leq 30$ & - & 1 & 4.2 \\
\hline
\end{tabular}

No CDC 3A, 4 or 5 complications occurred during follow-up. +, yes; +/-, unsure; -, no. ", Both non-procedure related and occurring after the same procedure. CDC, Clavien-Dindo classification.

OS and CSS of $87.5 \%$ and $100 \%$. Finally, we demonstrate the feasibility of metastasectomy as a procedure, with only two complications of CDC grade $3 \mathrm{~B}$ that were both nonprocedure related.

Patients in our series were treated within a 19-year long time span, in which clinical practice, imaging and therapies for metastatic UC have changed. Although treatment options for patients with metastatic UC have been limited during the last decades, CHT regimens have improved outcome in this patient population (7). Furthermore, the treatment landscape of metastatic UC has revolutionized with the advent of ICIs, which successfully target the PD-1/PD-L1 axis (11). Although these novel drugs deliver promising results, the ORRs remain limited. Therefore, selected patients could benefit from other treatment types such as a metastasectomy. Accumulating data show that survival can be improved with metastasectomy $(12,13)$. The benefit of metastasectomy has been demonstrated in other cancer types such as colorectal and renal cancer with a similar proliferation index as UC (14). However, patient selection is key. Patients with UC metastases who are amenable for metastasectomy are those with a relative long interval between primary surgery and UC recurrence, and with a good performance status. Our findings suggest that patients with pulmonary metastases benefit most from MDT. Moreover, better results were observed with small $(\leq 8 \mathrm{~mm})$ or solitary pulmonary lesions. Our lesion size cutoff of $8 \mathrm{~mm}$ was chosen based on statistical significance on univariate analysis. Other series have suggested a cutoff of $3 \mathrm{~cm}$ for pulmonary lesions, which could not differentiate 
significantly between patient groups in our cohort (15). Our results suggest that patients with primary UTUC involvement or hepatic metastases should be counseled cautiously for metastasectomy, as our results show that these patients had worse outcome after metastasectomy. The advantages and disadvantages of the procedure should be discussed with the patient during the attempt to increase their survival without putting the patient at risk of increased complications. Although we cannot draw strong conclusions from our retrospective cohort, our results can help clinicians when selecting appropriate patients for metastasectomy.

Our results confirm the findings of other retrospective series and suggest that patient selection could be based on number, location and size of metastasis (7). In this way, systemic platinum-based CHT and/or ICIs can be spared for further recurrences. Our reported 5-year OS of 51.4\% and median OS of 98.2 months are longer than reported by other series, however, within the limits reported by the meta-analysis of Patel et al. for metastasectomy in which various metastatic sites were included (6,16-18). Our 5-year OS of patients treated for any pulmonary metastasis (55.9\%) and for a solitary pulmonary metastasis $(87.5 \%)$, were in concordance with the results of pulmonary metastasis-only patients in the same meta-analysis (6). Moreover, we report a limited complication rate with a total of 12 complications for 24 metastasectomies. Only 2 complications were CDC grade $3 \mathrm{~B}$ and occurred after the same procedure and were non-procedure related, which illustrates the feasibility of this approach.

Till now, due to retrospective studies with limited number of cases, no strict criteria for the selection of patients for metastasectomy could be determined. Different centers have used their own eligibility criteria, with most of them being similar, such as good response to CHT, recurrence at the site of initial surgery, a solitary metastasis, feasibility of complete surgical resection of metastasis, no evidence of rapid progression at another metastatic location, or as a consolidative treatment with a period of disease stability (i.e., 3 months) between CHT and metastasectomy (17-19). In a similar concept, we meticulously selected our patients: most patients had a good performance status (ASA $\leq 2$ and ECOG $\leq 2$ ), had metastases that could be completely resected with negative surgical margins, and were willing to undergo aggressive treatment. Therefore, this cohort remains a highly selected subgroup of patients.

The difference in survival after metastasectomy between patients with primary BC or primary UTUC has not been demonstrated in all retrospective series $(7,20)$. The limited survival of patients with primary UTUC involvement in our cohort could be explained by the fact that both diseases are actually different disease entities with a different pattern of metastasis (21). The differences in primary disease being either primary BC, primary UTUC, or a combination of both could also have an impact on outcome, which could have biased our results.

This study is not devoid of limitations. The retrospective nonrandomized design of our study leads to an inherent selection bias, which could overestimate survival outcome compared with a real-life patient population, as the treated patients were deemed fit enough for metastasectomy. Heterogeneity in type of metastases, and type of metastasectomy procedure combined with a relatively small number of patients are the main limitations of our study. Due to the small number of patients, our statistical analyses were limited to univariate log-rank tests, which limits the strength of our conclusions.

Meticulous determination of the patients suitable for metastasectomy by a multidisciplinary team, procedures that were performed by experienced surgeons in each involved department, and employing a strict follow-up protocol for patients can be listed as strengths of this study. Additionally, only 2 patients received CHT prior to metastasectomy. Furthermore, there was no significant difference in OS, CSS or RFS between patients with or without CHT prior to metastasectomy, and patients that did not receive $\mathrm{CHT}$ prior to metastasectomy had a median OS of 98.2 months. As metastasectomy has been advocated as a consolidative treatment for patients who had (almost) complete response following CHT in previous series, our results can be interpreted as a hypothesis-generating study for the clinical benefit of metastasectomy alone, in the setting of oligometastatic UC.

Our study challenges the dogma that patients with metastatic UC are incurable and should be treated with systemic therapies only. We believe that the future lies in a multimodal approach in patients with oligometastatic disease that consists of a combination of surgery, radiotherapy and standard or new emerging systemic therapies that addresses the metastatic lesion(s) and also the presence of possible systemic disease. Future randomized clinical trials incorporating a molecular work-up using predictive biomarkers for treatment selection are urgently needed in the setting of oligometastatic UC.

\section{Conclusions}

Metastasectomy of oligometastatic UC recurrence is feasible 
and can achieve durable cancer control in a highly selected subgroup of patients. Our results suggest that patients with hepatic metastases, primary UTUC involvement or larger lesions $(>8 \mathrm{~mm}$ ) can be considered as poor candidates for metastasectomy, while patients with a solitary pulmonary lesion might benefit most. Metastasectomy should always be discussed within a multidisciplinary team. Well-designed multi-center randomized clinical trials should be performed to define patient selection criteria for metastasectomy in this setting.

\section{Acknowledgments}

$\mathrm{SJ}$ is a senior clinical investigator of the research foundation Flanders (FWO; number 1843518N).

Funding: None.

\section{Footnote}

Conflicts of Interest: All authors have completed the ICMJE uniform disclosure form (available at http://dx.doi. org/10.21037/tau-19-624). The authors have no conflicts of interest to declare.

Ethical Statement: The authors are accountable for all aspects of the work in ensuring that questions related to the accuracy or integrity of any part of the work are appropriately investigated and resolved. All procedures performed in this study working on human participants were in accordance with the ethical standards of the institutional research committee and with the 1964 Helsinki Declaration and its later amendments or comparable ethical standards. For this type of study, formal consent is not required.

Open Access Statement: This is an Open Access article distributed in accordance with the Creative Commons Attribution-NonCommercial-NoDerivs 4.0 International License (CC BY-NC-ND 4.0), which permits the noncommercial replication and distribution of the article with the strict proviso that no changes or edits are made and the original work is properly cited (including links to both the formal publication through the relevant DOI and the license). See: https://creativecommons.org/licenses/by-nc-nd/4.0/.

\section{References}

1. Global Burden of Disease Cancer Collaboration,
Fitzmaurice C, Allen C, et al. Global, regional, and national cancer incidence, mortality, years of life lost, years lived with disability, and disability-adjusted life-years for 32 cancer groups, 1990 to 2015: a systematic analysis for the Global Burden of Disease Study. JAMA Oncol 2017;3:524-48.

2. Stein JP, Lieskovsky G, Cote R, et al. Radical cystectomy in the treatment of invasive bladder cancer: long-term results in 1,054 patients. J Clin Oncol 2001;19:666-75.

3. Rink M, Lee DJ, Kent M, et al. Predictors of cancerspecific mortality after disease recurrence following radical cystectomy. BJU Int 2013;111:E30-6.

4. Robinson AG, Wei X, Vera-Badillo FE, et al. Palliative chemotherapy for bladder cancer: treatment delivery and outcomes in the general population. Clin Genitourin Cancer 2017;15:e535-41.

5. Resch I, Shariat SF, Gust KM. PD-1 and PD-L1 inhibitors after platinum-based chemotherapy or in first-line therapy in cisplatin-ineligible patients: Dramatic improvement of prognosis and overall survival after decades of hopelessness in patients with metastatic urothelial cancer. Memo 2018;11:43-6.

6. Patel V, Collazo Lorduy A, Stern A, et al. Survival after metastasectomy for metastatic urothelial carcinoma: a systematic review and meta-analysis. Bladder Cancer 2017;3:121-32.

7. Abufaraj M, Dalbagni G, Daneshmand S, et al. The role of surgery in metastatic bladder cancer: a systematic review. Eur Urol 2018;73:543-57.

8. Horwich A, Babjuk M, Bellmunt J, et al. EAU-ESMO consensus statements on the management of advanced and variant bladder cancer-an international collaborative multi-stakeholder effort: under the auspices of the EAU and ESMO Guidelines Committeest. Ann Oncol 2019;30:1697-727.

9. Dindo D, Demartines N, Clavien PA. Classification of surgical complications: a new proposal with evaluation in a cohort of 6336 patients and results of a survey. Ann Surg 2004;240:205-13.

10. Mitropoulos D, Artibani W, Graefen M, et al. Reporting and grading of complications after urologic surgical procedures: an ad hoc EAU guidelines panel assessment and recommendations. Eur Urol 2012;61:341-9.

11. Massari F, Di Nunno V, Cubelli M, et al. Immune checkpoint inhibitors for metastatic bladder cancer. Cancer Treat Rev 2018;64:11-20.

12. Hellman S, Weichselbaum RR. Oligometastases. J Clin Oncol 1995;13:8-10. 
13. Weichselbaum RR. The 46th David A. Karnofsky Memorial award lecture: oligometastasis-from conception to treatment. J Clin Oncol 2018;36:3240-50.

14. Bartlett EK, Simmons KD, Wachtel H, et al. The rise in metastasectomy across cancer types over the past decade. Cancer 2015;121:747-57.

15. Luzzi L, Marulli G, Solli P, et al. Long-Term Results and prognostic factors of pulmonary metastasectomy in patients with metastatic transitional cell carcinoma. Thorac Cardiovasc Surg 2017;65:567-71.

16. Abe T, Matsumoto R, Shinohara N. Role of surgical consolidation in metastatic urothelial carcinoma. Curr Opin Urol 2016;26:573-80.

17. Siefker-Radtke AO, Walsh GL, Pisters LL, et al. Is there a role for surgery in the management of metastatic urothelial cancer? The M. D. Anderson experience. J Urol 2004;171:145-8.

Cite this article as: Muilwijk T, Akand M, Van der Aa F, Dumez H, De Meerleer G, Van Raemdonck D, De Leyn P, Van Poppel H, Albersen M, Joniau S. Metastasectomy of oligometastatic urothelial cancer: a single-center experience. Transl Androl Urol 2020;9(3):1296-1305. doi:10.21037/tau-19624
18. Lehmann J, Suttmann H, Albers P, et al. Surgery for metastatic urothelial carcinoma with curative intent: the German experience (AUO AB 30/05). Eur Urol 2009;55:1293-9.

19. Abe T, Kitamura H, Obara W, et al. Outcome of metastasectomy for urothelial carcinoma: a multiinstitutional retrospective study in Japan. J Urol 2014;191:932-6.

20. Moschini M, Xylinas E, Zamboni S, et al. Efficacy of Surgery in the Primary Tumor Site for Metastatic Urothelial Cancer: Analysis of an International, Multicenter, Multidisciplinary Database. Eur Urol Oncol 2020;3:94-101.

21. Green DA, Rink M, Xylinas E, et al. Urothelial carcinoma of the bladder and the upper tract: disparate twins. J Urol 2013;189:1214-21. 

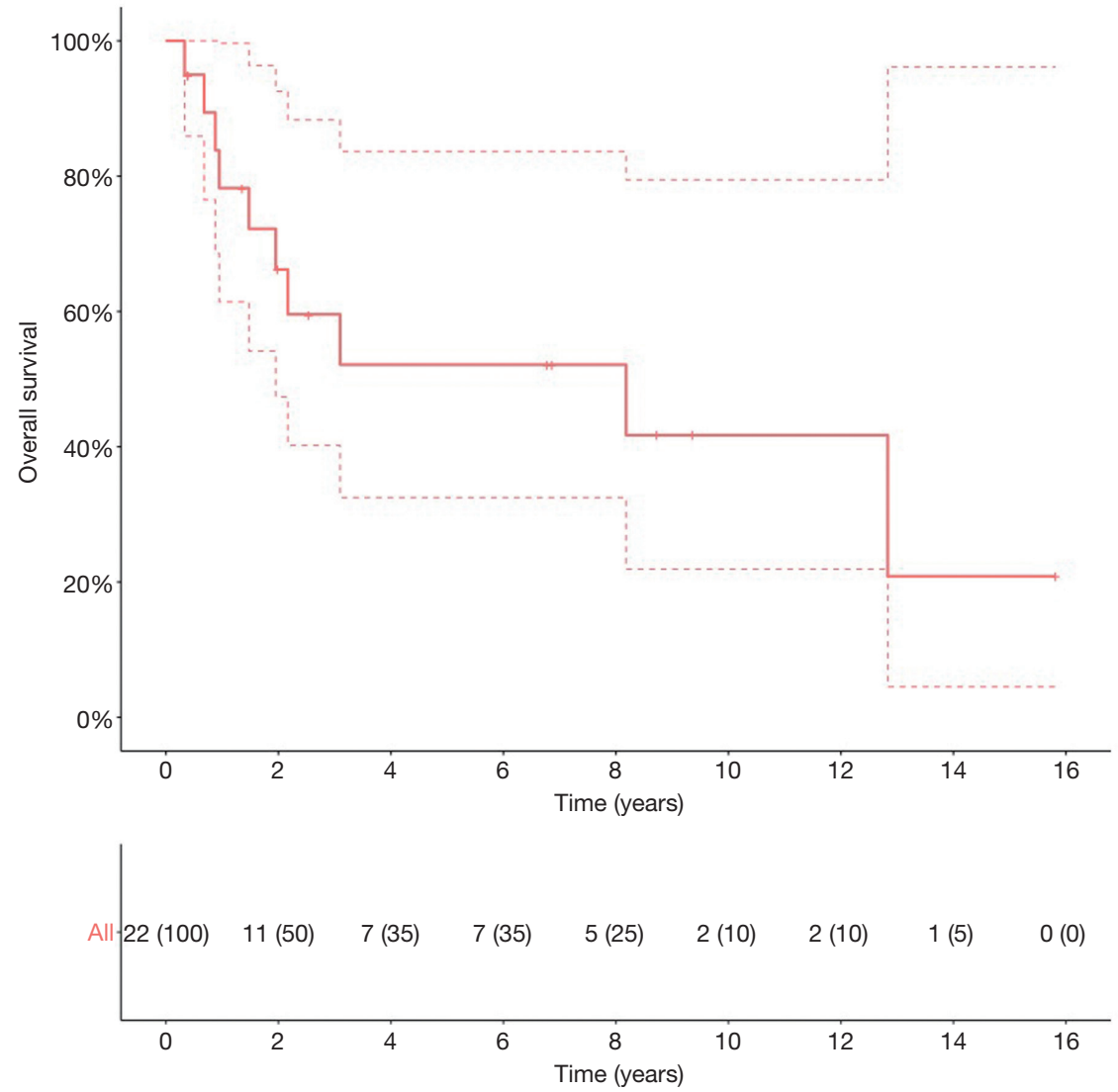

Figure S1 OS of all patients ( $\mathrm{n}=20)$ that did not receive any CHT prior to metastasectomy with a median OS of 98.2 months (IQR: 23.4NR). Dotted lines are the CI. OS, overall survival; CHT, chemotherapy; IQR, interquartile range; NR, not reached; CI, confidence interval. 

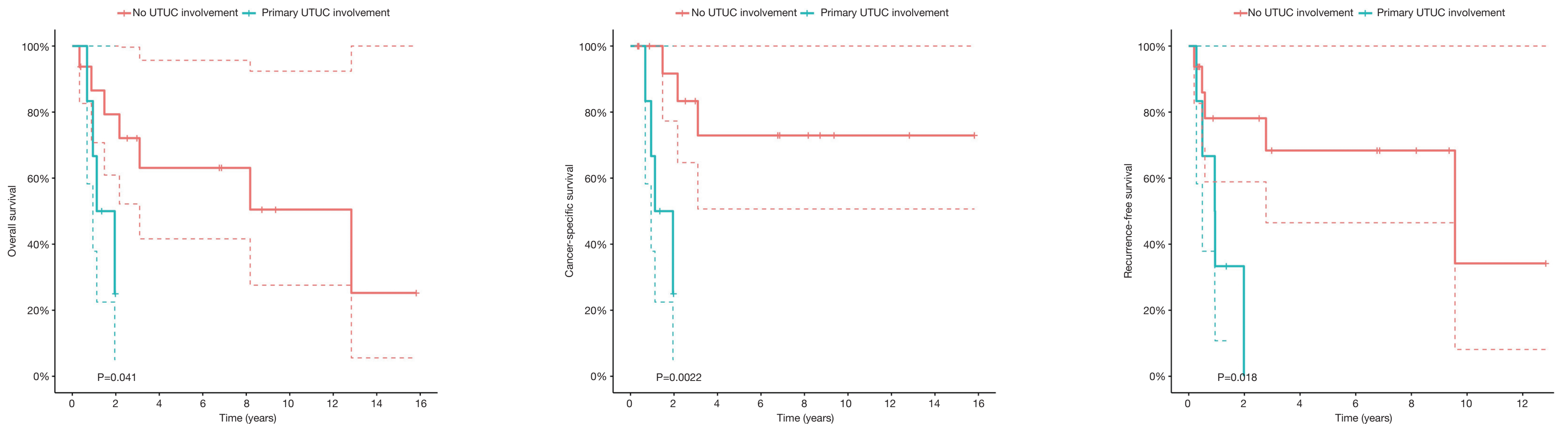

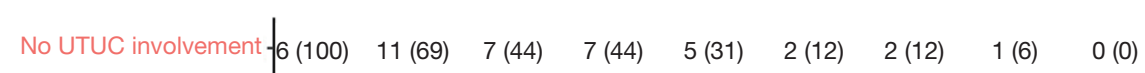
Primary UTUC involvement \begin{tabular}{ccccccccccc}
$6(100)$ & $0(0)$ & $0(0)$ & $0(0)$ & $0(0)$ & $0(0)$ & $0(0)$ & $0(0)$ & $0(0)$ \\
\hline 0 & 2 & 4 & 6 & 8 & 8 & 10 & 12 & 14 & 16
\end{tabular}

$\mathrm{D}$

+ No liver $\mathrm{M}++$ Liver $\mathrm{M}+$

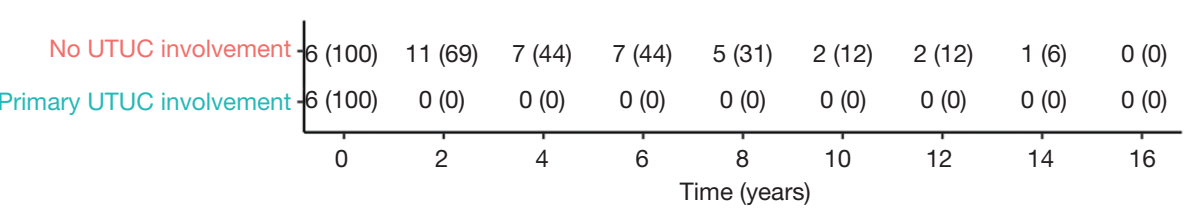

$\begin{array}{lllllll}\text { No UTUC involvement } 16(100) & 9(56) & 6(38) & 6(38) & 4(25) & 1(6) & 1(6)\end{array}$ Primary UTUC involvement \begin{tabular}{cccccccc}
$6(100)$ & $0(0)$ & $0(0)$ & $0(0)$ & $0(0)$ & $0(0)$ & $0(0)$ \\
\hline 0 & 2 & 4 & 6 & 8 & 10 & 12
\end{tabular}

+ No liver $\mathrm{M}++$ Liver $\mathrm{M}+$

+ No liver $\mathrm{M}++$ Liver $\mathrm{M+}$
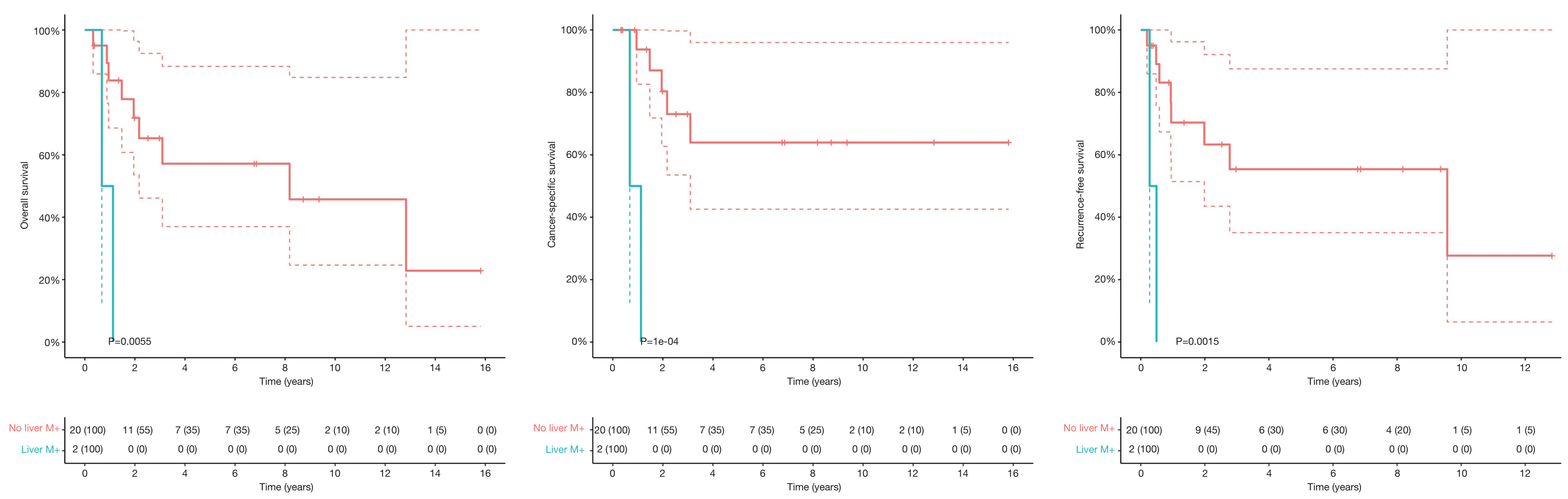

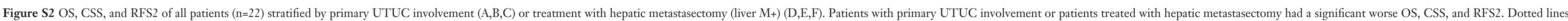
are the CI. OS, overall survival; CSS, cancer-specific survival; RFS2, secondary recurrence-free survival; UTUC, upper tract urothelial cancer; CI, confidence interval. 


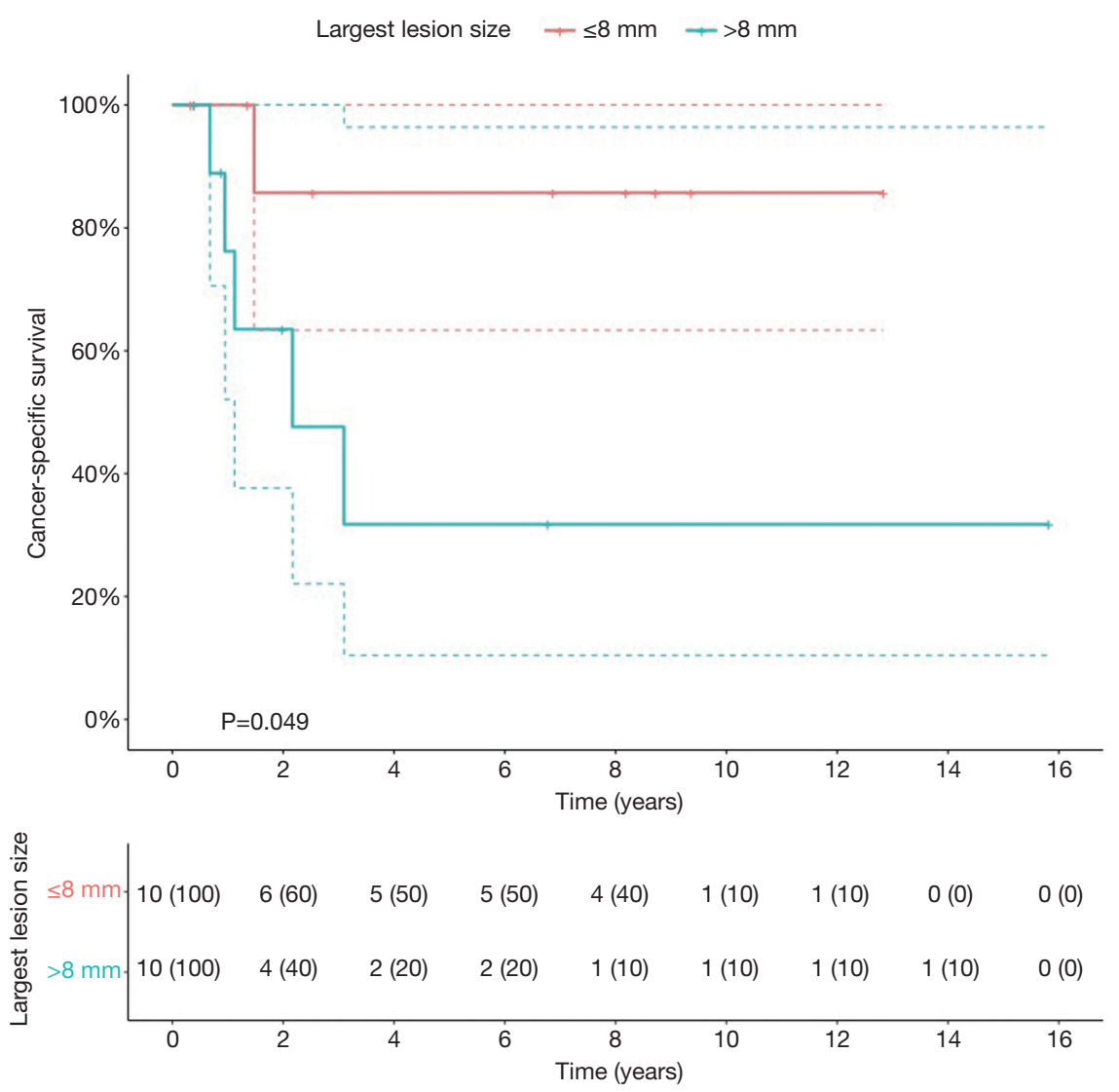

Figure S3 CSS of patients ( $\mathrm{n}=20)$ with available data regarding tumor size stratified by lesion size: $\leq 8 v s$. $>8 \mathrm{~mm}$. Patients with a lesion size $>8 \mathrm{~mm}$ had a significant worse CSS. Dotted lines are the CI. CSS, cancer-specific survival; CI, confidence interval.

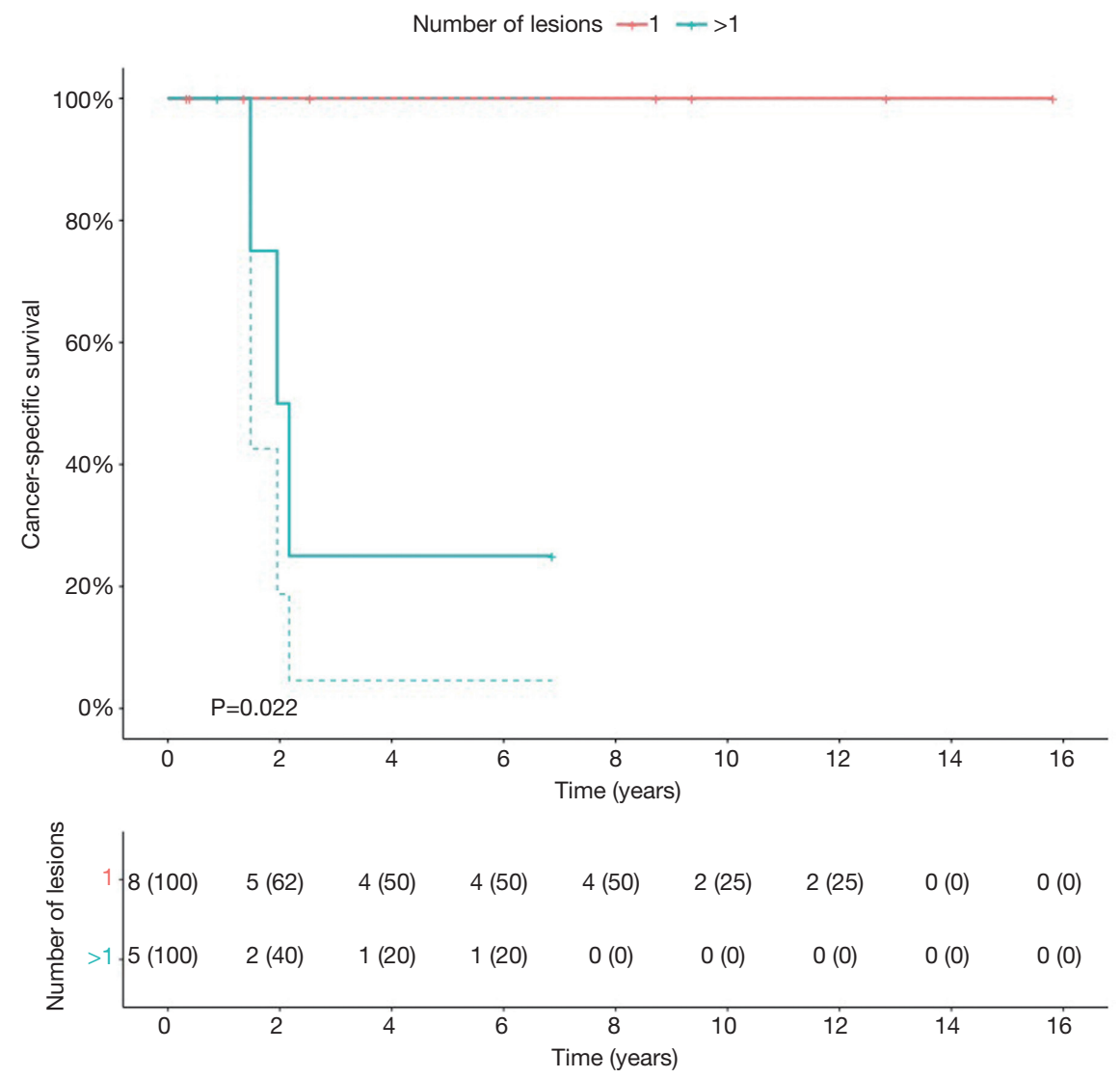

Figure S4 CSS of patients treated with pulmonary metastasectomy $(\mathrm{n}=13)$ stratified by number of lesions: solitary $v s$. $>1$. Patients with more than one lesion had a significant worse CSS. Dotted lines are the CI. CSS, cancer-specific survival; CI, confidence interval. 
Table S1 Type of metastasectomy procedures ( $\mathrm{n}=24)$ and number of complications per type of metastasectomy procedure $(\mathrm{n}=12)$

\begin{tabular}{|c|c|c|c|c|c|c|}
\hline Metastatic site & Procedure & Type of surgery & No & $\%$ & $\begin{array}{c}\text { No of } \\
C D C \leq 2\end{array}$ & $\begin{array}{c}\text { No of } \\
C D C \geq 3\end{array}$ \\
\hline \multirow[t]{2}{*}{ Pulmonary } & Wedge resection & Thoracoscopic & 8 & 33.3 & 3 & 0 \\
\hline & & Open & 2 & 8.3 & 0 & 0 \\
\hline Loco-regional & Abdominoperineal resection & Open & 1 & 4.2 & 1 & 0 \\
\hline \multirow[t]{2}{*}{ Hepatic } & Segmentectomy & Laparoscopic & 1 & 4.2 & 0 & 0 \\
\hline & & Open & 1 & 4.2 & 0 & 0 \\
\hline Renal transplant & Renal transplantectomy & Open & 1 & 4.2 & 1 & 2 \\
\hline Total & - & - & 24 & - & 10 & 2 \\
\hline
\end{tabular}

CDC, Clavien-Dindo classification; LND, lymph node dissection. 